\title{
Teaching Video NeuroImages: Anti-IgLON5 Disease
}

\section{A Long-Course Presentation With a Response to Immunotherapy}

Javier Villacieros-Álvarez, MD, Carlos Manuel Ordás, MD, Gustavo Torres-Gaona, MD, Ana Díez-Barrio, MD, Cristina Prieto-Jurczynska, MD, and Carles Gaig, PhD

Neurology ${ }^{\circledR}$ 2021;96:e2901-e2902. doi:10.1212/WNL.0000000000011381

A 74-year-old woman presented with a 5-year history of progressive gait instability with recurrent falls, dysphonia, dysphagia, and sleep problems, which included insomnia, breathing difficulties, and complex vocalizations during sleep (talking, singing, laughing). Examination showed bilateral ptosis, upward gaze palsy, hoarseness, dysprosody, and gait instability (video 1). No parkinsonism, chorea, or dementia was detected.

Brain MRI and PET as well as CSF measures were unremarkable. Video-polysomnography revealed a disorganized sleep architecture but not parasomnia. Anti-IgLON5 antibodies were positive in CSF and serum by cell-based assay of HEK cells transfected with IgLON5 and immunohistochemistry on rat brain. Human leukocyte antigen genotyping detected HLADQB1 ${ }^{*} 05: 01$.

Immunotherapy with IV methylprednisolone $1 \mathrm{~g} / \mathrm{d}$ for 5 days and immunoglobulins resulted in improvement of gait and speech over a month (video 1). A second course of immunoglobulins provided clinical stability.

Anti-IgLON5 disease represents a paradigm of autoimmune neurodegeneration with core features of a specific sleep disorder, bulbar symptoms, and gait abnormalities. ${ }^{1}$ Its recognition is important because some cases respond to immunotherapy. ${ }^{2}$

\section{Study Funding}

No targeted funding reported.

\section{Disclosure}

The authors report no disclosures relevant to the manuscript. Go to Neurology.org/N for full disclosures.

\section{Correspondence} Dr. Villacieros-Álvarez jvillacieros90@gmail.com

MORE ONLINE

- Video

$\rightarrow$ Teaching slides

lww.com/WNL/B301

From the Department of Neurology (J.V.-Á., C.M.O., G.T.-G., A.D.-B., C.P.-J.), Hospital Universitario Rey Juan Carlos, Móstoles; Department of Neurology (J.V.-Á., C.M.O., G.T.-G., A.D.-B., C.P.-J.), Hospital Universitario Infanta Elena, Valdemoro; Department of Neurology (J.V.-Á., C.M.O., G.T.-G., A.D.-B., C.P.-J.), Hospital General de Villalba, Collado Villalba, Madrid; and Department of Neurology (C.G.), Hospital Clinic, Barcelona, Spain.

Go to Neurology.org/N for full disclosures. 


\section{Appendix Authors}

\begin{tabular}{|c|c|c|}
\hline Name & Location & Contribution \\
\hline $\begin{array}{l}\text { Javier } \\
\text { Villacieros- } \\
\text { Álvarez, MD }\end{array}$ & $\begin{array}{l}\text { Hospital Universitario Rey } \\
\text { Juan Carlos; Hospital } \\
\text { Infanta Elena; Hospital } \\
\text { General de Villalba, Madrid, } \\
\text { Spain }\end{array}$ & $\begin{array}{l}\text { Designed and conceptualized } \\
\text { the study, major role in } \\
\text { acquisition of data, drafted the } \\
\text { manuscript for the intellectual } \\
\text { content, literature review }\end{array}$ \\
\hline $\begin{array}{l}\text { Carlos } \\
\text { Manuel } \\
\text { Ordás, MD }\end{array}$ & $\begin{array}{l}\text { Hospital Universitario Rey } \\
\text { Juan Carlos; Hospital Infanta } \\
\text { Elena; Hospital General de } \\
\text { Villalba, Madrid, Spain }\end{array}$ & $\begin{array}{l}\text { Major role in acquisition of } \\
\text { data, critical review of the } \\
\text { manuscript for intellectual } \\
\text { content }\end{array}$ \\
\hline $\begin{array}{l}\text { Gustavo } \\
\text { Torres- } \\
\text { Gaona, MD }\end{array}$ & $\begin{array}{l}\text { Hospital Universitario Rey } \\
\text { Juan Carlos; Hospital Infanta } \\
\text { Elena; Hospital General de } \\
\text { Villalba, Madrid, Spain }\end{array}$ & $\begin{array}{l}\text { Major role in acquisition of } \\
\text { data, critical review of the } \\
\text { manuscript for intellectual } \\
\text { content }\end{array}$ \\
\hline $\begin{array}{l}\text { Ana Díez- } \\
\text { Barrio, MD }\end{array}$ & $\begin{array}{l}\text { Hospital Universitario Rey } \\
\text { Juan Carlos; Hospital Infanta } \\
\text { Elena; Hospital General de } \\
\text { Villalba, Madrid, Spain }\end{array}$ & $\begin{array}{l}\text { Major role in acquisition of } \\
\text { data, critical review of the } \\
\text { manuscript for intellectual } \\
\text { content }\end{array}$ \\
\hline
\end{tabular}

Appendix (continued)

\begin{tabular}{lll}
\hline Name & Location & Contribution \\
\hline $\begin{array}{l}\text { Cristina } \\
\text { Prieto- } \\
\begin{array}{l}\text { Jurczynska, } \\
\text { MD }\end{array}\end{array}$ & $\begin{array}{l}\text { Hospital Universitario Rey } \\
\text { Infanta Elena; Hospital } \\
\text { General de Villalba, Madrid, } \\
\text { Spain }\end{array}$ & $\begin{array}{l}\text { Major role in acquisition of } \\
\text { data, critical review of the } \\
\text { manuscript for intellectual } \\
\text { content }\end{array}$ \\
\hline $\begin{array}{l}\text { Carles Gaig, } \\
\text { PhD }\end{array}$ & $\begin{array}{ll}\text { Hospital Clínic, Barcelona, } \\
\text { Spain }\end{array}$ & $\begin{array}{l}\text { Major role in acquisition of } \\
\text { data, critical review of the } \\
\text { manuscript for intellectual } \\
\text { content }\end{array}$ \\
& & \\
\hline
\end{tabular}

\section{References}

1. Gaig C, Graus F, Compta Y, et al. Clinical manifestations of the anti-IgLON5 disease. Neurology 2017;88:1736-1743.

2. Cabezudo-García P, Mena-Vázquez N, Estivill Torrús G, Serrano-Castro P. Response to immunotherapy in anti-IgLON5 disease: a systematic review. Acta Neurol Scand 2020;141:263-270. 


\section{Neurology}

\section{Teaching Video NeuroImages: Anti-IgLON5 Disease: A Long-Course Presentation With a Response to Immunotherapy}

Javier Villacieros-Álvarez, Carlos Manuel Ordás, Gustavo Torres-Gaona, et al. Neurology 2021;96;e2901-e2902 Published Online before print December 22, 2020

DOI 10.1212/WNL.0000000000011381

This information is current as of December 22, 2020

\section{Updated Information \&} Services

References

Subspecialty Collections

Permissions \& Licensing

Reprints including high resolution figures, can be found at: http://n.neurology.org/content/96/23/e2901.full

This article cites 2 articles, 1 of which you can access for free at: http://n.neurology.org/content/96/23/e2901.full\#ref-list-1

This article, along with others on similar topics, appears in the following collection(s):

All Clinical Neurology

http://n.neurology.org/cgi/collection/all_clinical_neurology Autoimmune diseases

http://n.neurology.org/cgi/collection/autoimmune_diseases

Information about reproducing this article in parts (figures,tables) or in its entirety can be found online at:

http://www.neurology.org/about/about_the_journal\#permissions

Information about ordering reprints can be found online:

http://n.neurology.org/subscribers/advertise

Neurology ${ }^{\circledR}$ is the official journal of the American Academy of Neurology. Published continuously since 1951, it is now a weekly with 48 issues per year. Copyright @ 2020 American Academy of Neurology. All rights reserved. Print ISSN: 0028-3878. Online ISSN: 1526-632X.

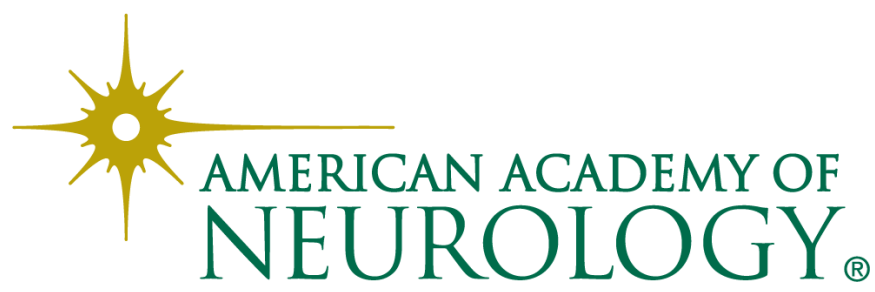

